\title{
Complications of anterior cervical discectomy and fusion using recombinant human bone morphogenetic protein-2
}

\author{
Rahul Vaidya $\cdot$ Julia Carp $\cdot$ Anil Sethi $\cdot$ Stephen Bartol $\cdot$ \\ Joseph Craig · Clifford M. Les
}

Received: 27 May 2006/Revised: 11 January 2007 / Accepted: 27 February 2007/Published online: 27 March 2007

(C) Springer-Verlag 2007

\begin{abstract}
The use of bone morphogenetic protein-2 (rhBMP-2) in spinal fusion has increased dramatically since an FDA approval for its use in anterior lumbar fusion with the LT cage. There are several reports of its use in transforaminal lumbar interbody fusion, posterolateral fusion, and anterior cervical fusion. Reports on adverse effects of rhBMP-2 when used in spinal fusion are scarce in literature. An Institutional Review Board approved retrospective study was conducted in patients undergoing anterior spinal fusion and instrumentation following diskectomy at a single center. Forty-six consecutive patients were included. Twenty-two patients treated with rhBMP-2 and PEEK cages were compared to 24 in whom allograft spacers and demineralized bone matrix was used. Patients filled out Cervical Oswestry Scores, VAS for arm pain, neck pain, and had radiographs preoperatively as well at every follow up visit. Radiographic examination following surgery revealed end plate resorption in all patients in whom rhBMP-2 was used. This was followed by a period of new bone formation commencing at 6 weeks. In contrast, allograft patients showed a progressive blurring of end plate-allograft junction. Dysphagia was a common complication and it was significantly more frequent and
\end{abstract}

R. Vaidya $(\bowtie) \cdot$ A. Sethi

Department of Orthopedics, Detroit Receiving Hospital,

University Health Center, 4201 St. Antoine Boulevard 6A, Detroit, MI 48201, USA

e-mail: rahvaidya@hotmail.com

J. Carp · S. Bartol · C. M. Les

Department of Orthopedics, Henry Ford Hospital,

2799 West Grand Boulevard K-12, Detroit, MI 48202, USA

J. Craig

Department of Radiology, Henry Ford Hospital,

2799 West Grand Boulevard K-12, Detroit, MI 48202, USA more severe in patients in whom rhBMP-2 was used. Post operative swelling anterior to the vertebral body on lateral cervical spine X-ray was significantly larger in the rhBMP2 group when measured from 1 to 6 weeks after which it was similar. These effects are possibly due to an early inflammatory response to rhBMP-2 and were observed to be dose related. With the parameters we used, there was no significant difference in the clinical outcome of patients in the two groups at 2 years. The cost of implants in patients treated with rhBMP-2 and PEEK spacers was more than three times the cost of allograft spacers and demineralized bone matrix in 1, 2, and 3-level cases. Despite providing consistently good fusion rates, we have abandoned using rhBMP-2 and PEEK cages for anterior cervical fusion, due to the side effects, high cost, and the availability of a suitable alternative.

Keywords Bone morphogenetic protein-2 - Cage . Anterior cervical fusion - Allograft bone

\section{Introduction}

Cervical disc disorders such as radiculopathy, stenosis with myelopathy, foraminal stenosis, disc herniation, and degenerative disc disease are commonly treated by anterior cervical discectomy and fusion surgery [2, 19, 23]. Fusion after discectomy is performed in order to limit motion, maintain disc height, foraminal height, and anatomic cervical lordosis at the intervertebral segment. The autograft techinique has historically been the most common procedure since its introduction in the 1950 s by Cloward, Smith, and Robinson [2, 23]. Patients that choose an autograft have a $90-95 \%$ successful fusion rate [23]. Complications of autograft harvest include graft site pain, infection, 
fracture, bleeding, and damage to the lateral femoral cutaneous nerve [23]. The use of allograft bone for cervical interbody fusion has become commonplace in areas where it is readily available, controlled by standard procurement, sterilization, and storage [1]. Once accepted by the body it is slowly transformed into new living bone and incorporated as a functional unit [19]. This technique eliminates the need for a second operative site and reduces the risk of infection [19].

Bone morphogenetic proteins (BMP) are osteoinductive proteins included in the superfamily of transforming growth factor-beta (TGF- $\beta$ ) [7, 8]. Bone morphogenetic proteins were identified several decades ago by Marshall Urist at UCLA, following evidence of bone growth in decalcified animal bone matrix [20]. Since then, BMP extracted and purified from cadaver bones has been used to accomplish bone formation [21,22]. The earliest reports of the clinical use of human BMP extracted from demineralized bone were in segmental bone defects and nonunions $[9,10]$. Human BMP extracted from allografts is of limited quantites. Bone morphogenetic proteins have been produced through recombinant methods to obtain unlimitied quantities [17, 21]. Through the use of recombinant genetic technology, a set of proteins, designated rhBMP-1 through rhBMP-9, have been produced. One of these recombinant proteins, rhBMP-2, was found to promote new bone and cartilage growth [8]. In 2002 the Food and Drug Agency in the United States approved the use of rhBMP-2 for anterior fusion when placed in an LT cage after an FDA trial which showed efficacy and safety (Medtronic Sofamor Danek). In the last 5 years researchers have published significant findings on rhBMP-2 compared to other bone graft alternatives for fusion. Studies indicate that patients that received rhBMP-2 obtained solid fusions without major adverse events [2-6, 12, 13, 15]. With such optimistic results in anterior lumbar interbody fusion, the use of rhBMP-2 has been expanded by surgeon preference to other spine fusion techniques. Several articles have focused on the use of rhBMP-2 in cervical fusion $[2,3]$, transforaminal lumbar interbody fusion (TLIF) [12], or posterolateral fusion [4]. When used for interbody fusion rhBMP-2 must be placed with a structural spacer to maintain disc height. It has been used with titanium cages, allograft bone dowels, and recently reported polyetheretherketone (PEEK) cages. PEEK is a nonresorbable semicrystalline aromatic polymer that is used to create a structural spacer [3] and is utilized in multiple implants. The purpose of this study is to evaluate the clinical and radiographic outcomes and cost differential in patients undergoing anterior cervical discectomy fusion and instrumentation (ACDFI) with rhBMP-2 (INFUSE, Medtronic Sofamor Danek, USA) and PEEK cages with our standard treatment of allograft spacers (MTF Synthes Spine, USA) and demineralized bone matrix.

\section{Materials and methods}

Study design

An IRB approved retrospective study was performed on 46 consecutive patients undergoing primary elective anterior cervical discectomy and fusion between February 2002 and August 2004. The first 12 patients had cervical fusions using allograft interbody spacers (MTF Synthes Spine) with demineralized bone matrix supplemented with an anterior locking plate (CSLP-Synthes Spine, USA). The next 22 patients had cervical fusions using rhBMP-2 with a PEEK cage supplemented with an anterior locking plate (Zephyr plate Medtronic Sofamor Danek, USA). The last 12 patients had cervical fusions with the allograft spacers, demineralized bone matrix and plate fixation. We included only primary patients scheduled for anterior cervical discectomy and fusion with either radiculopathy or myelopathy with a cause amenable in our hands to ACDFI. Patients with revisions, trauma, tumors, or better served by posterior surgery or combined surgery were excluded. This study thus included 46 patients who underwent primary elective anterior cervical discectomy and fusion. Twenty two consecutive patients with PEEK spacers and rhBMP-2 compared to 24 patients with allograft spacers and demineralized bone matrix all supplemented with an anterior locking plate.

\section{Patient data}

\section{Allograft group}

The indication for surgery in the allograft group was cervical disc disease and radiculopathy in 20 patients and cervical disc disease causing stenosis with radicular or myelopathic findings in four patients. There were 10 male and 14 female patients with an average age of 48 years (range 30-69). There were 11 single-level fusions, 10 twolevel fusions, and 3 patients with three-level fusions (24 patients 40 levels). The mean follow up was 28.03 months (range 16-42).

\section{RhBMP-2 group}

The indication for surgery in the rhBMP group was cervical disc disease with radiculopathy in 19 patients, and stenosis with myelopathic or radicular findings in 3 patients. There were 7 males and 15 female patients in the 
rhBMP-2 group with average age of 50 years (range 2970). There were 8 one-level fusions, 9 two-level fusions and 4 patients with three-level fusions (22 patients 38 levels). The mean follow up was 23.6 months (range 1926).

Anterior cervical diskectomy was performed using the standard anterior exposure. Levels were identified with C-arm guidance, self retaining retractors and a Caspar Vertebral distracter were used to aid in the diskectomy. Osteophytectomy was performed where required for neural decompression. The endplates were curreted to remove cartilage but were not violated with a high speed drill. Standard sizers were used with both implants and the appropriate allograft spacer or PEEK cage with rhBMP-2 were impacted into place. The rhBMP-2 sponges were prepared following the recomended technique and a dosage of $1 \mathrm{mg}$ of InFUSE $^{\mathrm{TM}}$ was used per level of fusion. Anterior locking plates were placed and the wound closed with a 1/8 inch hemovac drain in all cases. There was no bone graft harvesting in any case. Post operative care included a removable soft collar for comfort in all cases.

Patients were followed clinically at immediate post-op, 2, 6 weeks, 3, 6 months, 1 year and at latest follow up. Their Cervical Oswestry Scores, Arm and Neck VAS for pain, and a pain diagram were recorded. Standard digital radiographs were performed preoperatively and at every follow up appointment (stored on the electronic public acess computer system EPACS). Patients were questioned about dysphagia, hoarse voice and any other difficulties at every visit. Dysphagia was classified as mild (some discomfort), moderate (difficulty swallowing liquid, solids, or pills) and severe (inability to swallow fluids or solids, and requiring prolonged hospitalization).

Radiographs were assessed by three independant observers and one musculokeletal radiologist. They were assessed for prevertebral swelling, bone formation, subsidence and likelihood of fusion.

Soft tissue swelling was measured at C3 and at C6 as a digital line measurement from the front of the cervical spine to the edge of the soft tissue shadow. This measurement was standardized by the size of the plate.

$$
\begin{aligned}
& \text { Soft tissue swelling }(\mathrm{mm}) \times \frac{\text { Measured plate size }}{\text { Original plate size }} \\
& \quad=\mathrm{mm} \text { of swelling. }
\end{aligned}
$$

This ratio was then compared to the original post op film. To determine the accuracy of measurement we compared the size of the plate on ten different X-rays and found a variation of $<10 \%$. As there was no plate on the preoperative film we took the standard measurement from the digital radiograph realizing that there could be a $10 \%$ error in measurement. However, with numbers of 200-500\% increase in swelling the possibility of a $10 \%$ error appeared to be insignificant. The above measurements were done by using the Hospital Picture Archive and Communications System (PACS) (Stentor, S.F., CA, USA). The pixel resolution of the CR images was $0.12 \mathrm{~mm}$, making this the limiting accuracy in the measurements.

Bone formation was classified as no new bone, visible new bone, possible fusion and probable fusion for the rhBMP-2 group (Fig. 1). For the allograft group arthrodesis was assessed at the graft endplate junction and classified as not united, possibly united and probably united. CT scans were performed in any individual at 12 months if there was a concern of non union.

A series of two-way repeated-measures analyses of variance (Sigma Stat 2.03, SPSS, Chicago, IL, USA) were performed, looking at the effect of treatment (allograft vs. bmp) and time since surgery (pre-op, and 0.5, 1.5, 3, 6, 12, and 24 months postoperative) on the output variables (a) arm pain score, (b) neck pain score, and (c) Oswestry score. All output parameters were considered as continuous variables. Where appropriate (main effect alpha $=0.05$, interaction alpha $=0.10$ ), a Fisher least-squares difference post-hoc test was performed, with alpha $=0.05$.

Measurements of swelling anterior to the vertebral bodies were compared for the data points of pre-operative, and all post-operative visits between the two groups. A paired sample $t$ test was performed to generate a two tailed $P$ value with significance set at $<0.05$.

To compare the incidence of dysphagia and hoarse voice as a complication, the fisher exact test was used. Each data
Fig. 1 X-ray classification of rhBMP-2 fusion
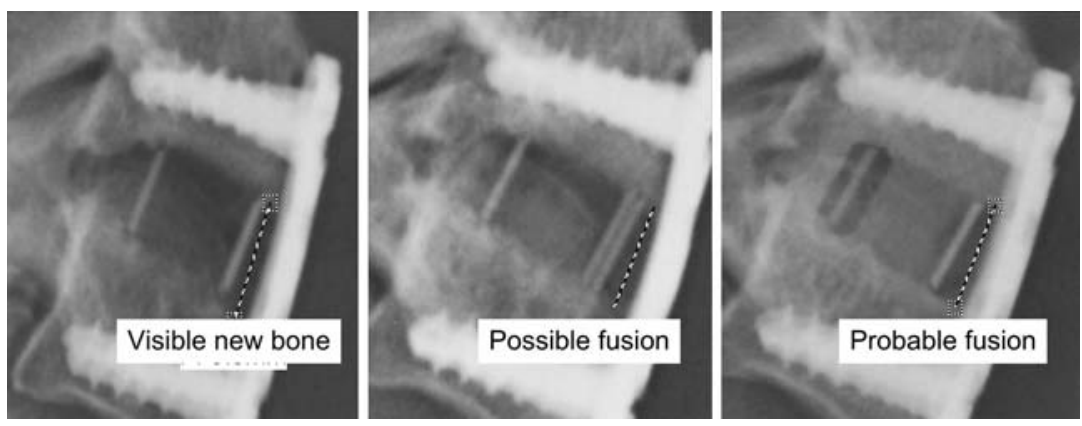
point on the post-operative follow up visits were compared. A two tailed $P$ was generated with significance set at $<0.05$. We did not statistically compare the severity of dysphagia between the two groups.

\section{Results}

Outcome measures

\section{Neck pain}

Neck pain scores were reviewed for 46 rhBMP-2 and allograft spacer patients pre-operatively and at 2, 6 weeks, 3, 6 months, 1 year and their latest follow up after surgery. A Visual Analogue scale was used and pain levels calculated as a percentage. Patients that received rhBMP-2 had average pain scores of 7.1, 4.8, 5.1, 4.8, 4.8, 3.9, and 2.6, respectively. Allograft patients had average pain scores of 8.5, 5.0, 4.2, 4.3, 4.2, 3.6, and 2.6, respectively (Fig. 2). There was no significant difference in pain scores between the rhBMP-2 and allograft spacer patients. There was significant improvement from pre-operative scores in both groups. There was no difference in pain scores between the rhBMP-2 and allograft spacer patients.

\section{Arm pain}

Arm pain scores were reviewed for 46 rhBMP-2 and allograft spacer patients pre-operatively and at 2, 6 weeks, 3 , 6 months, 1 year and the latest follow up post operatively. Again, pain scores ranked from one to ten, with ten being the most painful. Patients who received rhBMP-2 had average pain scores of 5.0, 3.6, 3.6, 3.1, 2.9, 2.4, and

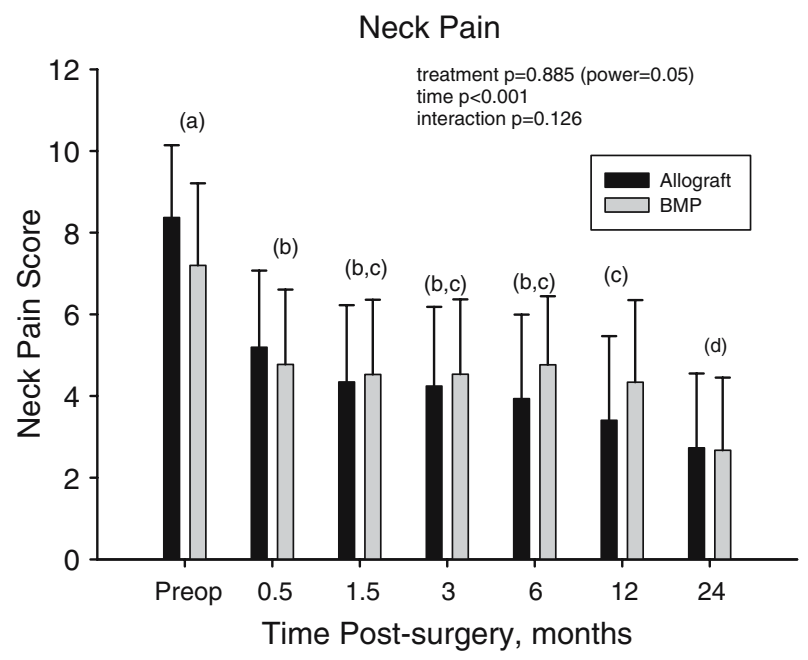

Fig. 2 Preoperative and follow up neck pain scores

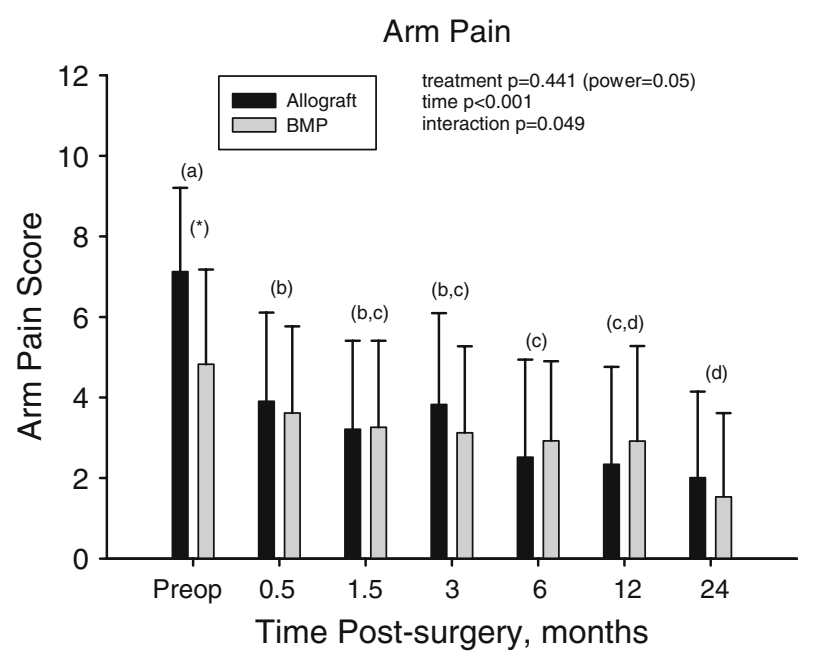

Fig. 3 Preoperative and follow up arm pain scores

1.3, respectively. Allograft spacer patients had average pain scores of 7.1, 3.8, 3.2, 4.0, 2.5, 2.4, and 1.8, respectively (Fig. 3). There was also no significant difference in pain scores between the rhBMP-2 and allograft spacer patients. There was improvement in both groups from their pre-operative scores.

\section{Oswestry scores}

Patients with rhBMP-2 had scores of 53.3, 56.9, 46.8, 45.1, $45.3,39.2$, and 28.8 respectively. Allograft spacer patients had scores of $60.4,58.8,54,50.9,39.3,32.8$, and 27.1 respectively. Again, there was no significant difference in Oswestry scores between the rhBMP-2 and allograft spacer patients (Fig. 4). There was improvement in both groups from their preoperative scores

Radiographic outcomes

\section{Arthrodesis and end plate erosion}

In the allograft group 23/24 patients achieved a diagnosis of probable fusion at latest follow up (39/40 levels). One patient with a 2 level fusion was diagnosed with a nonunion at 1 of 4 fusion surfaces on repeat exploration at 12 months, as she had ongoing neck pain and a nonunion was suspected. All patients in the rhBMP-2 group achieved a diagnosis of probable fusion at their latest follow up (38 levels). Interesting characteristics during the progression of fusion were noted in the group with rhBMP-2 and PEEK cages. We observed that there was end plate resorption in $100 \%$ of the levels where rhBMP-2 was used (Fig. 5). This phenomenon was first noted at 1.5 months post operatively, and lasted until 6 months. By 12 months all of the 38 levels were diagnosed as having a probable fusion (Fig. 6). 


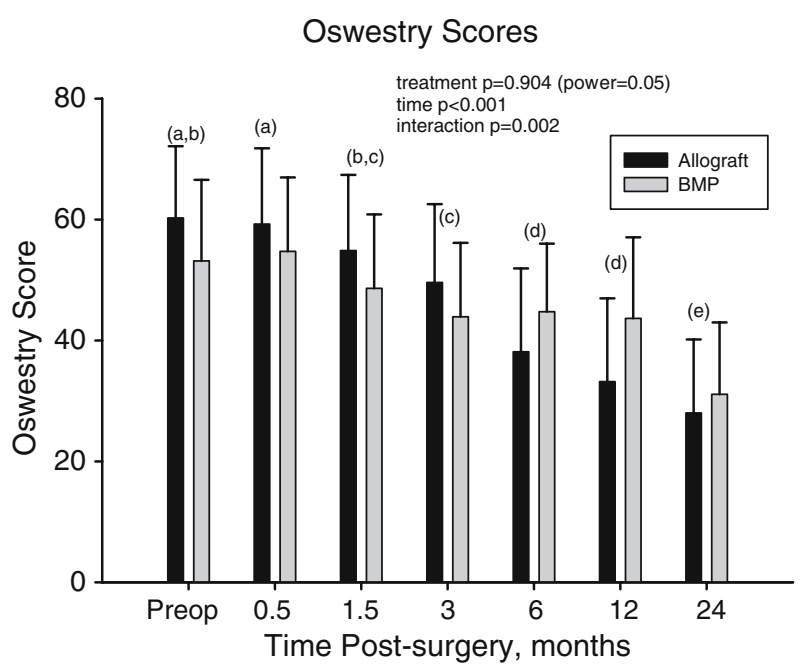

Fig. 4 Preoperative and follow up Oswestry disability index scores

\section{Distension anterior to the vertebral bodies}

Lateral digital radiographs were measured for distension anterior to the vertebral bodies at several time points. These points included, a pre and immediate post-operative assessment, as also at 2 and 6 weeks and 3, 6, 12, and 24 months following surgery. Both groups had similar preoperative values at $\mathrm{C} 3(5.0 \pm 1.7 \mathrm{~mm}$ rhBMP-2 vs. $5.0 \pm 1.5 \mathrm{~mm}$ allograft). All patients had distension of the soft tissue anterior to the cervical vertebrae during their post operative period (Fig. 7). When measured at C3 there was a significant difference in the swelling between the allograft and rhBMP-2 groups. These were altered immediate postoperatively $(15.7 \pm 7.8 \mathrm{~mm}$ rhBMP- 2 vs. $10.0 \pm 3.9 \mathrm{~mm}$ allograft $P=0.001$, and at 2 weeks $(11.8 \pm 3.7 \mathrm{~mm}$ rhBMP-2 vs. $8.3 \pm 3.0 \mathrm{~mm}$ allograft $P=0.01)$, and 6 weeks following surgery $(8.0 \pm 3.1$ rhBMP-2 vs. $5.9 \pm 3.1 \mathrm{~mm}$ allograft $P=0.03$ ) after which both groups returned to near pre-operative values (Fig. 8). There was no significant difference in the swelling when measured at the
C6 level. This increase in the soft tissue space anterior to the vertebrae was associated with difficulty in swallowing and led to one reoperation during the first post-operative week in the earliest cases. A wound infection was suspected and the patient was re-explored. However, there was no evidence of infection at surgery. Edematous tissue was seen, cultures of which were reported sterile. In hospital monitoring led to subsidence of symptoms.

\section{Complications}

\section{Dysphagia}

Thirty-eight patients were evaluated for dysphagia (20 rhBMP-2 and 18 allograft). Eighty-five percent of rhBMP2 patients and $56 \%$ of allograft patients reported difficulty with swallowing during the post-operative period (Fig. 9). There were statistically significant more patients with dysphagia in the rhBMP-2 group at 2 weeks (rhBMP-2 17/ 20, allograft $7 / 18$ fisher exact test $P=0.0092$ ) and 6 weeks after surgery (rhBMP-2 13/20, allograft 4/18 fisher exact test $P=0.0189$ ).

The number of levels of surgery affected the incidence of dysphagia. In single-level cases at 2 weeks it was $71 \%$ in patients with rhBMP-2 and only $13 \%$ in the allograft group $(P=0.069$, fisher exact test). In 2 and 3-level cases there was statistically significant less dysphagia in the allograft group at 6 weeks (allograft $40 \%$ to rhBMP-2 92\% $P=0.023$ fisher exact test) with 2 and 12 weeks also showing a similar trend, but not reaching statistical significance with our limited number of patients. At 2 year follow up $21 \%$ of patients still complained of mild dysphagia $($ rhBMP-2 $=20 \%$ and allograft $=22 \%)($ Fig. 8$)$.

\section{Hoarseness of voice}

There was no significant difference between rhBMP-2 and allograft spacer patients with respect to hoarseness of voice
Fig. 5 End plate resorption seen at 6 weeks
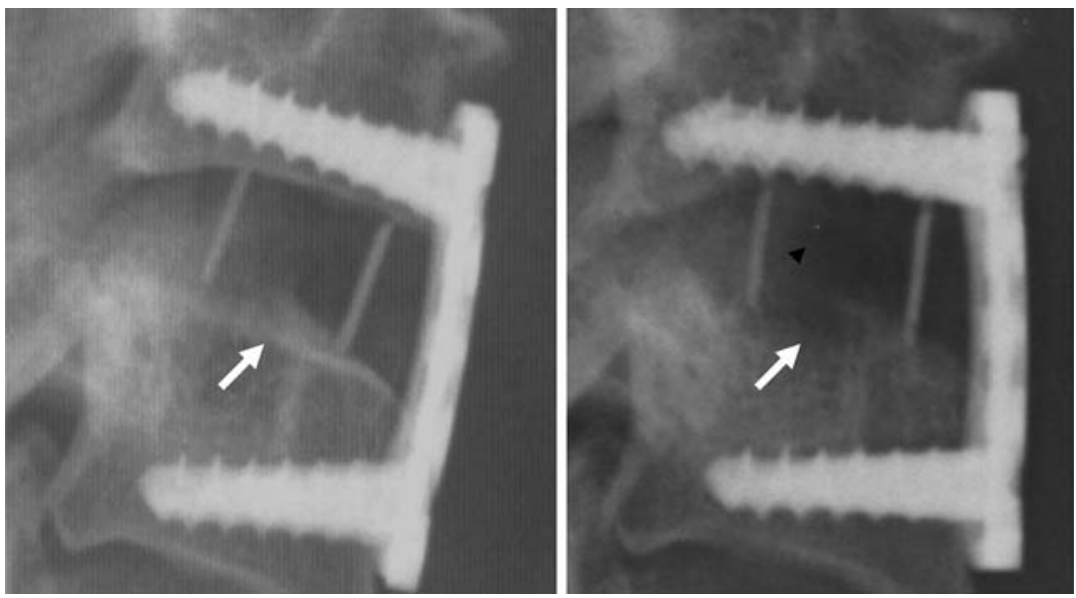
Fig. 6 Classification of fusion at each level versus time at follow up

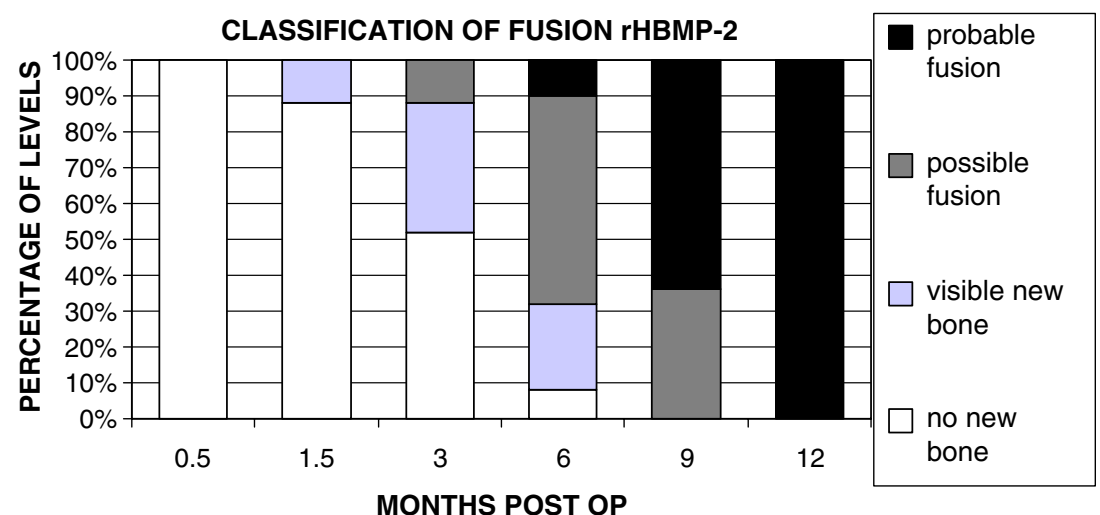

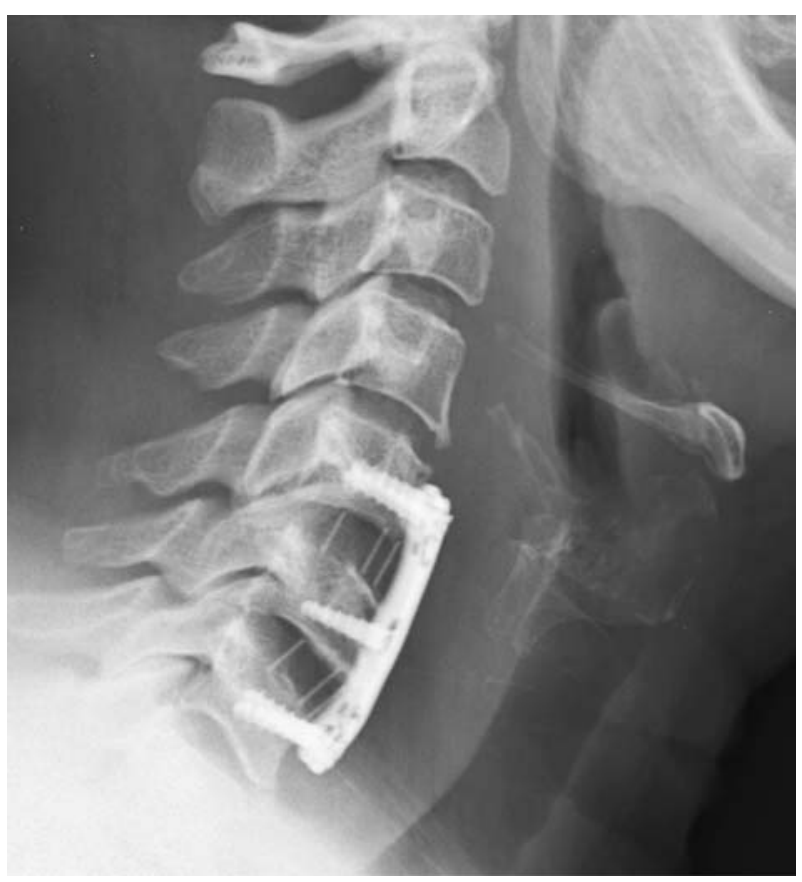

Fig. 7 Post operative swelling anterior to the vertebral bodies

Comparison of Average Swelling at C-3 as a Percentage in Patients with and without rhBMP-2

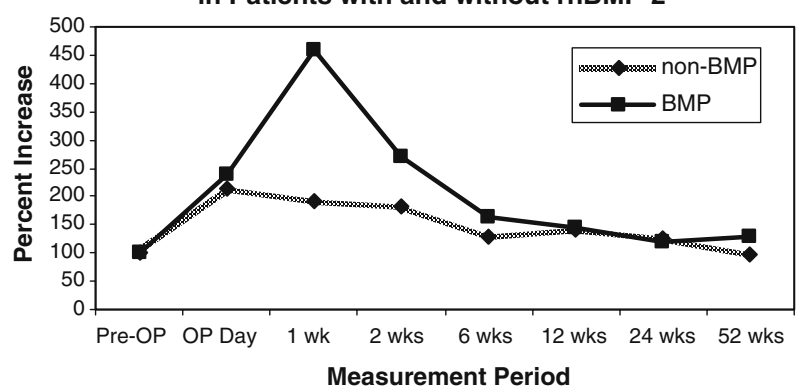

Fig. 8 Comparison of soft tissue distension at C3 in rhBMP-2 and allograft patients after surgery (fisher exact test). Sixty percent of rhBMP-2 patients and $62 \%$ of allograft spacer patients experienced post-operative voice hoarseness. One-level cases complained of hoarseness, nearly equally in allograft $(46 \%)$ and rhBMP-2 (50\%) group of patients. Two and three-level cases were also nearly similar (allograft $58 \%$ and rhBMP-2 $69 \%$ ) but the length of symptoms was considerably longer averaging 4 weeks until the symptom subsided in both groups with 2 or 3-levels. Overall 5 patients (3 allograft and 2 rhBMP-2) still complained of hoarseness at their last follow up. Two of these patients were 3-level fusions and three were 2-level fusions.

\section{Other complications and re-operations}

Three patients had re-operations during the follow up period. Wound exploration was performed in one case in the rhBMP-2 group as infection was suspected in the early post-operative period. Only edematous tissue was found and the patient improved steadily. One patient in the allograft group had a repeat surgery for a nonunion at 12 months. This was confirmed at surgery and treated with plate removal, a posterior fusion and instrumentation with relief of symptoms at 6 months. Finally, a third patient was operated at a lower level after having a PEEK cage with rhBMP- 2 which was healed at 15 months. One patient in the rhBMP-2 group continues to complain of neck pain in her upper cervical spine but refuses any repeat surgery and her radiographs appear to show probable fusion.

\section{Length of hospitalization}

On an average, rhBMP-2 patients remained in the hospital 0.66 days longer than allograft spacer patients. Patients with rhBMP-2 had a hospital stay ranging from one to nine days with an average stay of 2.95 days. This was affected by prolonged stay of three patients in the rhBMP-2 group with dysphagia which we described as severe. Allograft 
Fig. 9 a Dysphagia in patients with allograft. b Dysphagia in patients with rhBMP-2 and PEEK cages
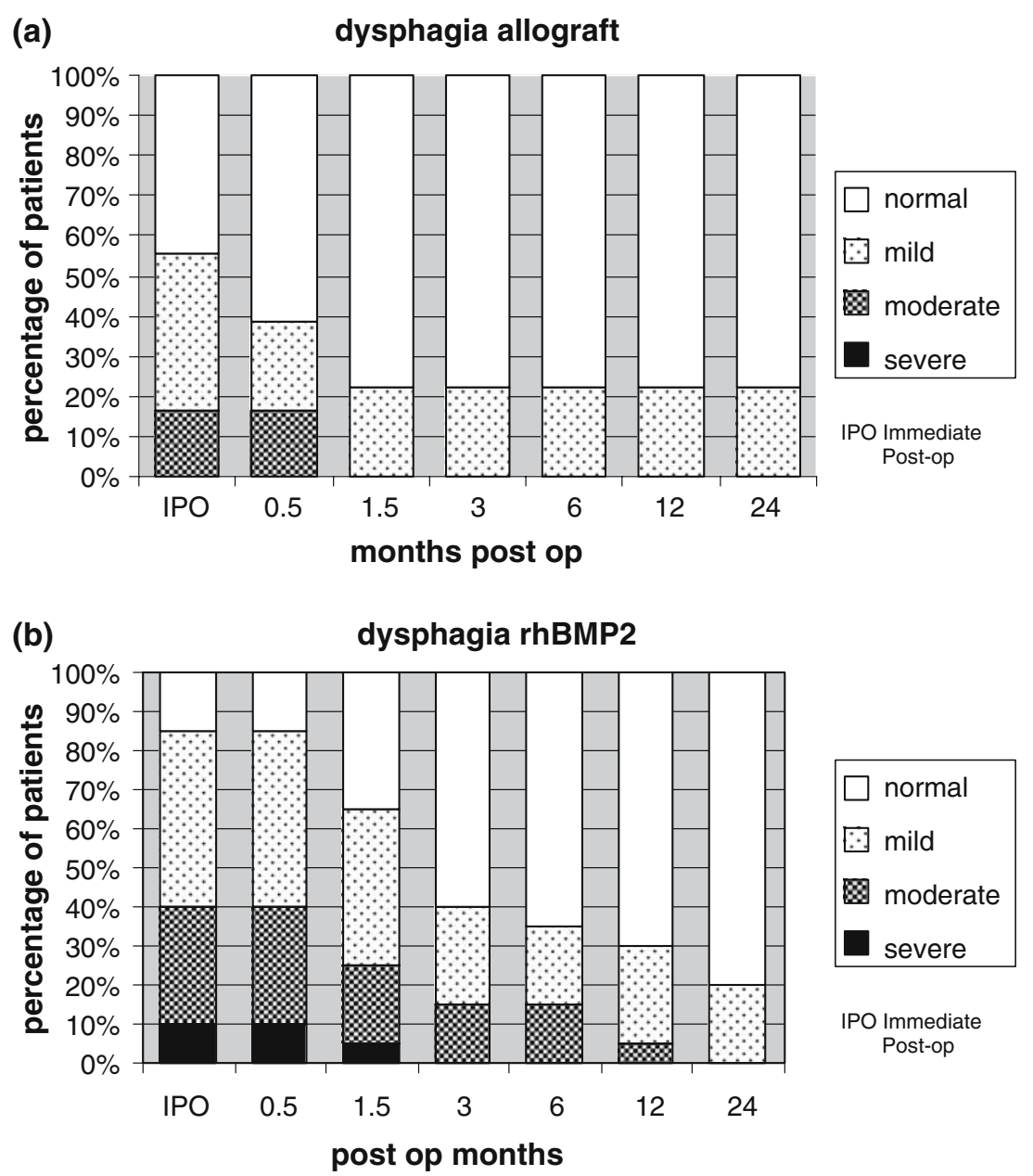

spacer patients had a hospital stay range from 1 to 6 days, with an average stay of 2.3 days.

\section{Cost of implants at surgery}

The average cost of implants using rhBMP-2 and allograft spacers were reviewed. Costs included cervical PEEK cages, rhBMP-2 (small), anterior cervical plate, allograft spacers, demineralized bone matrix, and cervical plates. The cost for surgical implants using rhBMP-2 with PEEK cages and an anterior cervical plate was $\$ 8,097$ for one-level surgery, $\$ 11,862$ for 2-level surgeries, and $\$ 15,588$ for 3-level surgeries. The cost of surgical implants using machined allograft spacers, demineralized bone matrix and an anterior cervical plate was: $\$ 2,096$ for 1-level surgery, $\$ 3,026$ for 2-level surgeries, and \$3,956 for 3-level surgeries. Although we did not track the operative time of surgery for each group, we did not expect any differences as neither procedure involves any extra bone graft harvesting or preparation of the implants by the surgeon. In the use of rhBMP-2 there is a nominal 35 min period that the circulating and operative nurse are involved during the preparation of the sponges.

\section{Discussion}

Recombinant human bone morphogenetic protein-2 is an osteoinductive protein significant for consistent new bone formation [23]. Through the use of rhBMP-2 in animal and a few human studies, it has been concluded that rhBMP-2 achieves successful fusion rates and better quality (stiffness) or quantity of bone mass with little disadvantage with its use [2, 4, 6, 14-17, 23]. Several papers have reported its use to achieve solid cervical fusions without major complications [2, 4, 6, 11-13, 15]. However, a recent study has documented the adverse effects following the use of rhBMP-2 with higher doses [18].

Interesting characteristics during the progression of fusion were noted in the group with rhBMP-2 and PEEK cages. We observed that there was end plate resorption in $100 \%$ of the levels where rhBMP-2 was used. This phenomenon was first noted at 1.5 months following surgery, and lasted until 6 months. This was a remarkable finding and was often mistaken as infection at the intervertebral level by the radiologist. End plate resorption was not seen in any of the allograft patients where a simple and pro- 
gressive blurring of the end plate allograft junction occurs. In patients with rhBMP-2 there was a progressive appearance of radiographic density at the intervertebral space that was felt to be the formation of new bone, seen from 6 weeks to 12 months after surgery (Fig. 1). By 12 months all of the 38 levels were diagnosed as having a probable fusion. From observing this consistent phenomenon it is our view that there is likely an early local inflammatory response to rhBMP-2 which is manifest as edema anterior to the vertebral bodies. This may be in conjunction with an early osteoclastic response manifest as resorption of endplates. It is followed by a progressive formation of a radiographic density which likely represents the formation of bone and is consistent with animal studies. Previous studies in animals may have missed this progression due to lack of serial radiographs early on in the fusion process. Also prior studies done with the titanium LT cage or allograft would certainly mask the early phenomenon of endplate resorption due to the titanium or hard bone contacting the endplate. Additionally studies without radiographs in the first 2 weeks may miss many of the findings described. We did not use CT scans to determine fusion in our patients unless we suspected a problem. This certainly would have been a more accurate marker of fusion but due to cost restraints it was not performed routinely. For this reason we called our end points probable fusion when clinical markers remained good and radiographs displayed the progressive formation of a density in the rhBMP-2 group. It is our view that this represented the formation of bone (Fig. 3). We used radiographic blurring of the endplates to diagnose a probable fusion in the allograft group. Patients that did not show persistent improvement in outcome scores were further evaluated with imaging studies. One patient in our study was diagnosed with a non union and had a reoperation.

Dysphagia is a common occurrence after anterior cervical disk surgery and is often persistent [18]. Patients that received rhBMP-2 had more difficulty swallowing after surgery. They also suffered this complication more severly and for a longer period of time. Three patients required prolonged hospitalization and one patient required a feeding tube for 6 weeks post operatively. This was accompanied by soft tissue distension anterior to the vertebral bodies noted on post-operative radiographs. It was significantly greater than that seen in the allograft patients. It is suggested that these effects are likely due to an early inflammatory local response to the rhBMP-2 as this was not nearly as evident in the allograft group. In the one patient re-explored for distension anterior to the vertebral bodies only edematous local tissue was seen. We observed this phenomenon to be dose related. Severe dysphagia was not observed in single-level cases with rhBMP-2 although they did have more difficulty swallowing than the allograft patients. The 2 and 3-level patients had more difficulty than the single-level patients treated with rhBMP-2 and statistically significant more difficulty than the 2 and 3-level cases with allograft.

With the limited number of patients in this study we observed that the two groups were fairly similar in age, gender, and pathology. The patients were not stratified or selected but were consecutive patients that were limited by their diagnosis. Pre-operatively there were subtle differences in neck pain, arm pain and Oswestry scores between the two groups. In the long term follow up differences in the scores persisted but they were not statistically significant.

The cost for implants in patients treated with rhBMP-2 was higher and since the final outcomes of the two groups were similar an increased expense cannot be justified. The length of hospital stay in the rhBMP-2 group was slightly longer due to the complication of dysphagia adding further expense to this procedure. If nonunions were considerably more likely in the allograft group there might be some justification in the use of rhBMP-2. However, our one nonunion (96\% fusion rate) with the use of allograft is in keeping with the literature. In our current practice we have abandonned the use of rhBMP-2 in cervical fusions due to the associated dysphagia, high cost of implants and the availability of a good alternative. In the future, we may reconsider this option if a smaller dose of rhBMP-2 leads to fusion with the same efficacy and lesser side effects. It may also be an option if it is available considerably cheaper, or if the safety of allograft procurement becomes suspect.

The limitations of this study include the fact that it is retrospective with a limited follow up period (23.6 and 28 months) and includes only 46 patients. A larger study may be able to show some subtle differences in outcome scores and other less prevalent complications. Additionally, using radiographs and clinical outcomes as the endpoint for a diagnosis of fusion is not optimal. Hence, the endpoint in this study is listed as probable fusion in both groups and not fusion itself. Although we do not suspect nonunions to present late after 2 years, it still remains a possibility and long term results are desirable.

\section{Conclusion}

The use of rhBMP-2 with PEEK cages and an anterior cervical plate in the dosage which we used, led to consistent fusion in the cervical spine. Early radiographic findings included end plate erosion and marked soft tissue swelling anterior to the vertebral bodies which was significantly greater than in the allograft cases. The radiographic events in the process of fusion led to probable fusion at 1 year in $100 \%$ of rhBMP- 2 cases. However, due to significantly increased incidence of dysphagia, higher 
cost, and good success with a cheaper alternative, we currently do not use PEEK cages and rhBMP-2 in anterior cervical spine fusion.

\section{References}

1. A guide from the The American Academy of Orthopedic Surgeons (AAOS) and American Association of Tissue Banks (AATB): What can you tell me about bone and tissue transplantation. Retrieved June 21, 2005 from http://www.aatb.org/ aaosinfo.htm

2. Baskin DS, Ryan P, Sonntag V, Westmark R, Widmayer MA (2003) A prospective, randomized, controlled cervical fusion study using recombinant human bone morphogenetic protein-2 with the CORNERSTONE-SR ${ }^{\mathrm{TM}}$ allograft ring and the ATLANTIS $^{\text {TM }}$ anterior cervical plate. Spine 28(12):1219-1224

3. Boakye M, Mummaneni PV, Garrett M, Rodts G, Haid R (2005) Anterior cervical discectomy and fusion involving a polyetheretherketone spacer and bone morphogenetic protein. J Neurosurg Spine 2:521-525

4. Boden SD, Kang J, Sandhu H, Heller JG (2002) Use of recombinant human bone morphogenetic protein-2 to achieve posterolateral lumbar spine fusion in humans: a prospective, Randomized clinical pilot trial 2002 Volvo Award in clinical studies. Spine 27(23):2662-2673

5. Burkus JK (2004) Bone morphogenetic proteins in anterior lumbar interbody fusion: old techniques and new technologies. J Neurosurg Spine 3:254-260

6. Burkus JK, Heim SE, Gornet MF, Zdeblick TA (2004) The effectiveness of rhBMP-2 in replacing autograft: an integrated analysis of three human spine studies. Orthopedics 27(7):723728

7. Celeste AJ, Iannazzi JA, Taylor RC, Hewick RM, Rosen V, Wang EA, Wozney JM (1990) Identification of transforming growth factor beta family members present in bone-inductive protein purified from bovine bone. Proc Natl Acad Sci USA 87(24):9843-9847

8. Hidehiro I, Sohei E, Mikio K, Yutaka T, Tetsuya K, Yohei Y, Kunio T (1999) Experimental spinal fusion with use of recombinant human bone morphogenetic protein 2. Spine 24(14):1402-1405

9. Johnson EE, Urist MR, Finerman GAM (1988) Bone morphogenetic protein augmentation grafting of resistant femoral nonunions. A preliminary report. Clin Orthop Relat Res 230:257-265

10. Johnson EE, Urist MR, Finerman GA (1988) Repair of segmental defects of the tibia with cancellous bone grafts augmented with human bone morphogenetic protein. A preliminary report. Clin Orthop Relat Res 236:249-257

11. Kleeman TJ, Michael Ahn U, Talbot-Kleeman A (2001) Laparoscopic anterior lumbar interbody fusion with rhBMP-2: a prospective study of clinical and radiographic outcomes. Spine 26(24):2751-2756

12. Mummaneni PV, Pan J, Haid RW, Rodts GE (2004) Contribution of recombinant human bone morphogenetic protein-2 to the rapid creation of interbody fusion when used in transforaminal lumbar interbody fusion. J Neurosurg Spine 1:19-23

13. Poynton AR, Lane JM (2002) Safety profile for the clinical use of bone morphogenetic proteins in the spine. Spine 27(16S):S40 S48

14. Sandhu HS (2003). Bone morphogenetic proteins and spinal surgery. Spine 28(15S):S64-S73

15. Sandhu HS, Khan SN (2003) Recombinant human bone morphogenetic protein-2: use in spinal fusion applications. J Bone Joint Surg 85-A(Suppl 3):89-95

16. Sandhu HS, Toth JM, Diwan AD, Seim HB, Kanim LEA, Kabo JM, Turner AS (2002) Histologic evaluation of the efficacy of rhBMP-2 compared with autograft bone in sheep spinal anterior interbody fusion. Spine 27(6):567-575

17. Schimandle JH, Boden SD, Hutton WC (1995) Experimental spinal fusion with recombinant human bone morphogenetic protein-2. Spine 20(12):1326-1337

18. Shields LB, Raque GH, Glassman SD, Campbell M, Vitaz T, Harpring J, Shields CB (2006) Adverse effects associated with high-dose recombinant human bone morphogenetic protein-2 use in anterior cervical spine fusion. Spine 31(5):542-547

19. Ullrich PF (1999) Anterior cervical spinal fusion surgery. Retrieved June 21, 2005 from http://www.spine-health.com/topics/ surg/overview/cervical/cerv03.html

20. Urist MR (1965) Bone: formation by autoinduction. Science 150:893-899

21. Wang EA, Rosen V, D'Alessandro JS, Bauduy M, Cordes P, Harada T, Israel DI, Hewick RM, Kerns KM, LaPan P et al (1990) Recombinant human bone morphogenetic protein induces bone formation. Proc Natl Acad Sci USA 87(6):2220-2224

22. Wang EA, Rosen V, Cordes P, Hewick RM, Kriz MJ, Luxenberg DP, Sibley BS, Wozney JM (1988) Purification and characterization of other distinct bone-inducing factors. Proc Natl Acad Sci USA 85(24):9484-9488

23. Zdeblick TA, Ghanayem AJ, Rapoff AJ, Swain C, Bassett T, Cooke ME, Markel M (1998) Cervical interbody fusion cages: an animal model with and without bone morphogenetic protein. Spine 23(7):758-765 